\title{
POLÍTICAS PÚBLICAS DE ATENÇÃO À SAÚDE DA MULHER NO TRATAMENTO DO CÂNCER DE MAMA EM FEIRA DE SANTANA - BA
}

\author{
Camila Curcino Santos ${ }^{1}$; Rita de Cássia Rocha Moreira ${ }^{2}$ \\ 1. Bolsista PROBIC/UEFS, Graduanda do curso de Enfermagem, Universidade Estadual de Feira de Santana, e-mail: \\ camiliacurcino@hotmail.com \\ 2. Orientadora, Departamento de Saúde, Universidade Estadual de Feira de Santana, e-mail: \\ ritahelio01@yahoo.com.br
}

PALAVRAS-CHAVE: Políticas Públicas em saúde; Câncer de mama; Saúde da Mulher.

\section{INTRODUÇÃO}

A atenção à saúde da mulher foi incorporada às políticas nacionais de saúde nas primeiras décadas do século XX, sendo limitada, nesse período, às demandas relativas à gravidez e ao parto. Com a evolução das mesmas, foi incluída a Atenção a mulher com câncer de mama. Essas políticas vêm sendo desenvolvidas no Brasil desde meados dos anos 1980 e foram impulsionadas pelo Programa Viva Mulher, em 1998 (BRASIL, 2004). O câncer de mama é o tipo mais comum entre as mulheres no mundo e no Brasil, com um risco estimado de 56,33 casos a cada 100mil mulheres para os anos de $2018 \mathrm{e}$ 2019 em nosso país (INCA, 2018). Portanto, a justificativa para a realização deste estudo, é de possibilitar uma análise da atenção fornecida a esse público, contribuindo com a produção científica da área, com a formação de acadêmicos dos cursos de saúde, além de estabelecer estratégias de capacitação dos profissionais da rede para o atendimento à saúde da mulher com o diagnóstico de câncer de mama. Teve como objetivo, compreender o processo de implementação das políticas públicas no tratamento do câncer de mama em mulheres atendidas na Unidade de Alta Complexidade no Município de Feira de Santana- Bahia, no período de julho de 2017 a julho de 2018.

\section{METODOLOGIA}

Estudo exploratório, qualitativo, teve como campo de estudo a Unidade de Alta Complexidade (UNACON) em Feira de Santana-BA no período de julho de 2017 a julho de 2018. Teve como questão de pesquisa: como tem se dado o processo de implementação das Políticas Públicas para a tratamento do câncer de mama nas Unidades de Alta Complexidade no Munícipio de Feira de Santana-Ba? Para coleta de dados, foi utilizado como instrumento, um roteiro de entrevista contendo 11 questionamentos. Como técnica de coleta de dados, foi utilizada a entrevista semiestruturada. Participaram do estudo 14 profissionais da unidade e coordenadores de serviço. Para a análise dos dados, foi utilizado o método de análise de conteúdo de Bardin (2011). O projeto ao qual este plano de Iniciação Científica (IC) está vinculado, foi aprovado pelo Comitê de Ética em Pesquisa da UEFS, parecer de Emenda versão 05 de número 2.386.774 de 19 de Novembro de 2017 e respeitou a Resolução 466/2012 do Conselho Nacional de Saúde, que determina as diretrizes e normas que regulamentam as pesquisas envolvendo seres humanos com assinatura do Termo de Consentimento Livre e Esclarecido (TCLE) por parte dos participantes da pesquisa. 


\section{RESULTADOS E DISCUSSÃO}

A Portaria $\mathrm{n}^{\circ}$ 874, de 16 de maio de 2013 institui a Política Nacional para a Prevenção e Controle do Câncer na Rede de Atenção à Saúde das Pessoas com Doenças Crônicas no âmbito do Sistema Único de Saúde (SUS). Tem como objetivos, promover, prevenir, diagnosticar, tratar, reabilitar e oferecer cuidados paliativos nas esferas nacionais, estaduais e municipais (BRASIL, 2013). O método de análise de conteúdo de Bardin (2011), foi a base para consolidar os resultados e discussões deste trabalho de IC. Na categoria analítica 01 - Fatores que dificultam a assistência ao câncer de mama; os entrevistados relataram a dificuldade que as mulheres têm para ter o diagnóstico precoce do câncer de mama na Atenção Básica (AB), em especial, pela desarticulação nas esferas da União, Estado e Município, o que eleva o atraso no tratamento. Afirmaram que há muitos pacientes que precisam realizar os exames, mas, devido a um número reduzido de cotas para os procedimentos específicos, acaba demorando na chegada na UNACON. Para Parada et al. (2008), persistem como nós críticos o acesso aos exames de rastreamento e as referências para diagnóstico e tratamento. É na $A B$ que o rastreamento para a enfermidade tem o seu início, porém, precisa da articulação com a Atenção Secundária e Terciária para que as mulheres acometidas por neoplasia da mama sejam assistidas de forma integral. Em relação ao que as pacientes têm encontrado como dificuldade para o início e manutenção do tratamento, os entrevistados relataram entraves para que a mulher possa agendar a primeira consulta com o oncologista e a realização dos exames de rotina. A deficiência na manutenção do tratamento se dá, por conta de questões socioeconômicas das usuárias, necessitando da assistência das prefeituras. A Portaria $\mathrm{n}^{\circ}$ 055, de 24 de fevereiro de 1999, dispõe sobre o Tratamento Fora de Domicílio (TDF), que envolve a garantia de transporte para tratamento e hospedagem, quando indicado (BRASIL, 1999; INCA, 2014). Fica evidente que existem leis que asseguram a garantia da permanência dos pacientes oncológicos no período do tratamento, porém, nem todos conseguem ter acesso aos benefícios. Para Sonobe, Buetto e Zago (2011), as usuárias desconhecem seus direitos e ficam dependentes, sujeitas a discriminação e menosprezadas pelo sistema. $\mathrm{Na}$ Categoria Analítica 02 - Necessidades de Atendimento e Condutas terapêuticas para o Câncer de Mama na Unidade de Alta complexidade, foi descrito pelos entrevistados, o problema da falta de exames para estadiamento da neoplasia. Porém, conforme a sua estrutura organizacional, está previsto que a UNACON não realiza exames diagnósticos e nem de estadiamento. Um entrevistado evidenciou o tamanho impróprio da unidade, o que dificulta o trabalho, resultando em atendimento inadequado às usuárias. $\mathrm{O}$ estudo apontou uma lacuna importante que é a falta de Mastologistas no serviço e fisioterapeutas para a assistência às mulheres com câncer de mama. Ficou evidenciado a deficiência da UNACON para a conduta de assistência às mulheres, em especial no que se refere ao cumprimento da Portaria 140, no item III que trata da garantia dos profissionais de ginecologia e mastologia No que se refere as condutas terapêuticas validadas pela comunidade científica para o tratamento do câncer de mama em mulheres, que não são custeados pelo SUS, e são consideradas importantes para o sucesso do tratamento, os entrevistados ponderaram sobre a existência de medicamentos para o câncer de mama e aumento de sobrevida dessas pacientes. Apesar 
de alguns relatos que apontaram o cerceamento de direitos das pessoas com câncer, um entrevistado relatou sobre a implementação da Política Nacional de Assistência Oncológica (PNAO) na qual, alguns medicamentos que não eram custeados pelo SUS passam a ser. Na Categoria Analítica 03 - Unidade de Alta Complexidade e os Serviço prestados no controle e tratamento do câncer de mama, foi relatado que a manutenção de equipamentos para o controle de tratamento câncer de mama funcionam de forma adequada e são submetidos a manutenção periódica de acordo com a Portara $n^{\circ} 140$, de 27 de fevereiro de 2014 (BRASIL, 2014b). Sobre o Sistema de Informação e Educação Permanente dos profissionais que atuam na UNACON, alguns entrevistados relataram não ter conhecimento do Registro Hospitalar de Câncer (RHC). Este registro tem sido descrito como um espelho que reflete o desempenho do corpo clínico em relação à assistência prestada aos pacientes entre outros (INCA, 2018). Sobre a existência de cursos periódicos realizados pelo SUS para capacitação de profissionais que atuam na área de oncologia, houve contradição nas respostas, nas quais os entrevistados relatavam que existiam cursos fornecidos pelo SUS, outros negaram e falaram da realização de Educação Continuada no serviço que existia para alguns, outros nunca tinham realizado. A UNACON deve promover meios para a realização de Educação Permanente e Continuada desses profissionais e o SUS em contrapartida deve fornecer meios de estudos e pesquisas para os profissionais, como forma de garantir uma atenção à saúde atualizada e adequada às mulheres com câncer de mama.

\section{CONSIDERAÇÕES FINAIS}

As Políticas Públicas para o Câncer de mama desde o seu surgimento, trouxe benefícios para a saúde de toda população, em especial a feminina, sendo ela a que mais sofre com a incidência do câncer de mama. Foi abordado as principais carências que a UNACON tem enfrentado diante dos recursos disponibilizados pelos SUS, as quais se referem, as possibilidades para tratamento integral e com protocolos atualizados que possam garantir a terapêutica adequada para as mulheres. $\mathrm{O}$ estudo permitiu reconhecer, que melhorias precisam ser realizadas na assistência ao câncer de mama, como a realização do diagnóstico precoce mais rápido, na Rede de Atenção Primária e Secundária, fortalecidas e regidas pelos princípios de universalidade, equidade e integralidade do SUS. Também defendemos a garantia do aumento no número de exames para se estabelecer o estadiamento da patologia, de forma que a mulher possa ter um melhor prognóstico. Deve ser garantido, o aperfeiçoamento nos tratamentos de adjuvância e paliativos para essas mulheres, vislumbrando a possibilidade de oferecer qualidade no atendimento. Este estudo apontou, a necessidade de ampliar o conhecimento dos profissionais que atuam na UNACON sobre o câncer de mama, além de estimular os profissionais da Atenção Básica a se articularem entre os setores para que não haja falhas na assistência. A assistência em oncologia deve ampliar o seu olhar para o fazer em saúde, na perspectiva de que, a cura e o controle da doença, devem permear uma assistência em defesa da qualidade de vida. Portanto, este estudo representa uma contribuição para a formação acadêmica de futuros profissionais enfermeiros, médicos, pesquisadores em saúde, além de estimular o aperfeiçoamento da equipe multidisciplinar da UNACON a manter o atendimento hora dispensado para Feira de Santana e cidade circunvizinhas. 


\section{REFERÊNCIAS}

BARDIN, L. Análise de conteúdo. Lisboa: Edições 70, 2011.

BRASIL, Portaria ${ }^{\circ}$ 55, 24 de fevereiro de 1999. Dispõe sobre a rotina do Tratamento

Fora de Domicilio no.Sistema Único de Saúde - SUS, com inclusão dos procedimentos específicos na tabela de procedimentos do Sistema de Informações Ambulatoriais do SIA/SUS e dá outras providências. Disponível em: < http://bvsms.saude.gov.br/bvs/saudelegis/sas/1999/prt0055_24_02_1999.html> Acesso em: 23 de julho de 2018.

BRASIL, Política nacional de atenção integral à saúde da mulher: princípios $e$ diretrizes/ Ministério da Saúde, Secretaria de Atenção à Saúde, Departamento de Ações Programáticas Estratégicas. - Brasília: Ministério da Saúde, 2004.

BRASIL, Portaria $\mathrm{n}^{\circ} 874$ de 04 de maio de 2013. Institui a Política Nacional para a Prevenção e Controle do Câncer na Rede de Atenção à Saúde das Pessoas com Doenças Crônicas no âmbito do Sistema Único de Saúde (SUS). Disponível em: < http://bvsms.saude.gov.br/bvs/saudelegis/gm/2013/prt0874_16_05_2013.html> Acesso em: 23 de julho de 2018.

BRASIL, Portaria $\mathrm{n}^{\mathrm{o}}$ 140, de 27 de fevereiro de 2014b. Redefine os critérios e parâmetros para organização, planejamento, monitoramento, controle e avaliação dos estabelecimentos de saúde habilitados na atenção especializada em oncologia e define as condições estruturais, de funcionamento e de recursos humanos para a habilitação destes estabelecimentos no âmbito do Sistema Único de Saúde (SUS). Disponível em: < http://bvsms.saude.gov.br/bvs/saudelegis/sas/2014/prt0140_27_02_2014.html> Acesso em: 23 de julho de 2018.

INCA. Coordenação Geral de Ações Estratégicas. Divisão de Comunicação Social. Direitos sociais da pessoa com câncer / Instituto Nacional de Câncer José Alencar Gomes da Silva. Coordenação Geral de Ações Estratégicas. Divisão de Comunicação Social. - 4a ed. - Rio de Janeiro: INCA, 2014. Disponível em:

<http://www2.inca.gov.br/wps/wcm/connect/d2afbb8048c5399da41bb67b3abc37df/Dir eitos+Sociais+da+pessoa+com+c\%C3\%A2ncer+4ed_WEB.pdf?MOD=AJPERES\&CA CHEID=d2afbb8048c5399da41bb67b3abc37df >. Acesso em: 27 jul. 2018.

INCA. Registro Hospitalar do Câncer. 2018 Disponível em:

<http://www.inca.gov.br/conteudo_view.asp?id=351>. Acesso em: 1 agosto 2018.

INCA. Estimativa 2018: Incidência de Câncer no Brasil / Instituto Nacional de Câncer José Alencar Gomes da Silva, Coordenação de Prevenção e Vigilância. Rio de Janeiro: PARADA, Roberto et al. A política nacional de atenção oncológica e o papel da atenção básica na prevenção e controle do câncer. Revista Aps, Si, v. 2, n. 11, p.199-206, jun. 2008. Disponível em: <https://aps.ufjf.emnuvens.com.br/aps/article/view/263/100>. Acesso em: 30 jul. 2018.

SONOBE, Helena Megumi; BUETTO, Luciana Scatralhe; ZAGO, Márcia Maria Fontão. O conhecimento dos pacientes com câncer sobre seus direitos legais. Revista da Escola de Enfermagem da Usp, [s.1.], v. 45, n. 2, p.342-348, abr. 2011. FapUNIFESP (SciELO). Disponível em:

<http://www.scielo.br/scielo.php?script=sci_arttext\&pid=S0080-62342011000200006>. Acesso em: 23 jul. 2018. 